\title{
Image Compression using DCT upon Various Quantization
}

\author{
Wael M. Khedr \\ Zagazig Univ., \\ Faculty of Science, \\ Math. Dept.Majmaah Univ., \\ College of Science in Zulfi,CSI Dept
}

\author{
Mohammed Abdelrazek \\ Zagazig Univ., \\ Faculty of Science, \\ Math. Dept.
}

\begin{abstract}
Discrete cosine transform (DCT) is a widely compression technique for converting an image into elementary frequency components. However, level of quality and compression is desired, scalar multiples of the JPEG standard quantization may be used. In this paper, DCT method was applied to compress image under various level of quality. Different quantization matrices of DCT's coefficients are used to improve level of quality and compression ratio of JPEG image.
\end{abstract}

\section{Keywords}

DCT , Image compression, Quantization, and PSNR

\section{INTRODUCTION}

Image compression is a technique to reduce the amount of data required for representing sampled digital images and therefore reduce the cost for storage and transmission $[2,4]$. Compression techniques are classified into lossless and lossy compression. In lossless compression techniques, the original image can be perfectly recovered from the compressed (encoded) image [2]. Whereas in lossy compression the reconstructed image from the compressed image is similar to the original image but not identical to it. The previous works on DCT [1] compression was using one quantization matrix, almost the jpeg standard one. In this paper we proposed DCT method based on various quantization matrices of DCT's coefficients. Determined quantization matrix level is related to the standard deviation of DCT's coefficient blocks. We compared between traditional DCT methods with the proposed method. We found that results showed more compression ratio and better quality for our method than traditional one. The rest of this paper is organized into four sections. The second section explains the traditional DCT. The third section explains the Quantization. The proposed algorithm is described in fourth section. Finally the experimental result obtained in this paper in the last section.

\section{DISCRETE COSINE TRANSFORM}

Discrete Cosine Transform (DCT) $[1,3,8]$ is one of many transforms that takes its image and transforms it into a linear combination of weighted basis functions. These basis functions are commonly the frequency, like sine waves. 2-D DCT of an image is defined as in Eq.(1).

$$
\begin{aligned}
& D(i, j)=\frac{1}{\sqrt{2}} C(i) C(j) \sum_{x=0}^{N-1} \sum_{y=0}^{N-1} I(x, y) \cos \left[\frac{(2 x+1) i \pi}{2 N}\right]\left[\frac{(2 x+1) j \pi}{2 N}\right] \\
& \text { Where: } C(u)=\left\{\begin{array}{ll}
\frac{1}{\sqrt{2}} & \text { if } u=0 \\
1 & \text { if } u>0
\end{array},\right.
\end{aligned}
$$

and $\mathrm{I}(\mathrm{x}, \mathrm{y})$ is gray level of the $\mathrm{x}, \mathrm{y}$ element of the image represented by the matrix I, $\mathrm{N}$ is the size of the block.

\section{QUANTIZATION}

Quantization is the process of reducing the number of possible values of a quantity, thereby reducing the number of bits needed to represent it [10].

\subsection{Jpeg Standard Quantization}

The JPEG standard quantization matrix of the DCT [9] coefficients with a quality level of 50 provides high compression and excellent decompressed image quality. We can get on different level of quality and compression ratio by determine a suitable quantization matrix for DCT coefficients.

$$
Q_{50}=\left[\begin{array}{cccccccc}
16 & 11 & 10 & 16 & 24 & 40 & 51 & 61 \\
12 & 12 & 14 & 19 & 26 & 58 & 60 & 55 \\
14 & 13 & 16 & 24 & 40 & 57 & 69 & 56 \\
14 & 17 & 22 & 29 & 51 & 87 & 80 & 62 \\
18 & 22 & 37 & 56 & 68 & 109 & 103 & 77 \\
24 & 35 & 55 & 64 & 81 & 104 & 113 & 92 \\
49 & 64 & 78 & 87 & 103 & 121 & 120 & 101 \\
72 & 92 & 95 & 98 & 112 & 100 & 103 & 99
\end{array}\right]
$$

From standard Quantization matrix in Eq.2, we can obtain on different quantization $\mathrm{Qn}$ levels by using following equation:

$$
Q_{n}= \begin{cases}\left(\frac{100-n}{50}\right) Q_{50} & , \quad n>50 \\ \left(\frac{50}{n}\right) Q_{50} & , \quad n<50\end{cases}
$$

Where $\mathrm{n}$ is level of Quality. When $\mathrm{n}$ is greater than 50, we get on less compression and high quality and when $\mathrm{n}$ is less than 50 we get on more compression and less quality. The following figure(1) describes quantization matrices yields quality levels of 50, 90, and 10 .

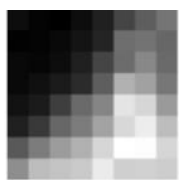

(a) $Q 50$

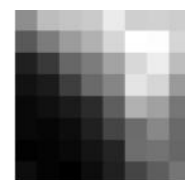

(b) $Q 90$

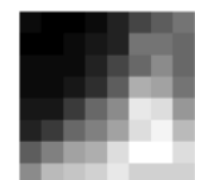

(c) Q10
Figure 1 : Quantization levels of 50,90 , and 10

\subsection{Quantization Upon Variance}

The variance and standard deviation measure how far a set of numbers is spread out, (zero indicates that all the values are identical). In images, if the gray values of block $(8 \times 8)$ are identical (ex : one color) then the variance equal zero and the value increase with the extent of the number of different colors in the block. 
Figure (2) shows Papers image, the maximum of standard deviation for all non-overlap blocks $(8 \times 8)$ is 1.9812 and its minimum is 0.0067 .

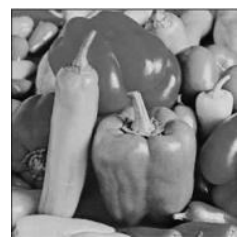

Figure 2 : Peppers Image
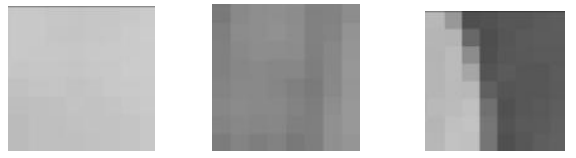

(a)Low Variance

(b) Medium variance

(c)

High

Variance

Figure 3 : Different $(8 \times 8)$ blocks from Pepper image.

Figure (3.a) shows no important details so it can be quantized with low quality and high compression, also Figure (3.b) shows some details but not that much so we can keep medium quality and compression. And in Figure (3.c), there are details and edges which should be clear which required high quality and less compression ratio.

\section{HUFFMAN CODING}

Huffman coding $[12,13]$ is the variable length coding, wherein the length of each code word is a function of frequency of occurrence of a symbol. JPEG standard defines the Huffman tables which are used to encode output of differential and run-length process. Huffman coding is a form of statistical coding which attempts to reduce the amount of bits required to represent a string of symbols. The algorithm accomplishes its goals by allowing symbols to vary in length. Shorter codes are assigned to the most frequently used symbols, and longer codes to the symbols which appear less frequently in the string (that's where the statistical part comes in). Code word lengths are no longer fixed like ASCII .Code word lengths vary and will be shorter for the more frequently used characters.

\section{PROPOSED METHOD}

A remarkable and highly useful feature of JPEG process is that in quantization step. Varying levels of image compression and quality are obtained through selection of specific quantization matrices. This enables the user to decide on quality levels ranging from 1 (poorest) to 100 (Best Quality).The selection of Quantization matrix is depended on the variance of each block in image.

\subsection{Algorithm Steps}

The steps of proposed method are:

1) The image is divided into $(8 \times 8)$ blocks of pixels.

2) DCT is applied to each block from right to left, top to bottom.

3) The variances of each DCT's coefficients blocks are calculated.

4) The blocks are divided into three classes based on value of their variance [high , medium , low]
5) Each class (high , medium , low) is compressed through corresponding Quantization matrix (Q90，Q50，Q10) respectively.

6) The compressed blocks that constitute the image is encoded with Huffman code and stored in a drastically reduced amount of space.

7) Finally the image is reconstructed through decompression using IDCT (Inverse Discrete Cosine Transform)

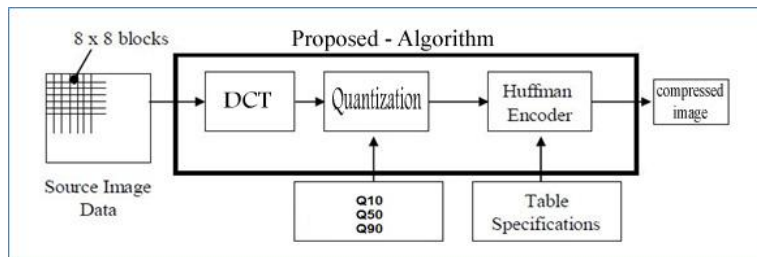

Figure 4 : Algorithm steps

\subsection{Experimental Results}

Experiments are performed on three gray images as given in figure(5) and the reconstruction image is shown in figure(6).

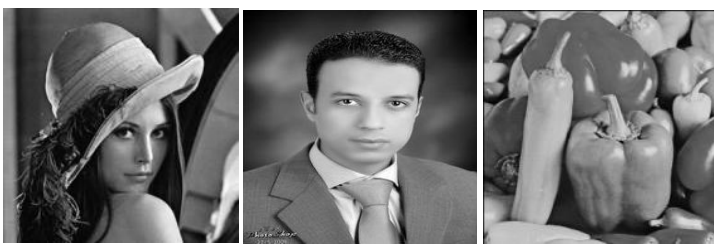

Figure 5. Reconstruction images Lena, Mohammed and Peppes..

To measure the quality of the image after reconstruction we used the mean square error MSE which defined as

$$
M S E=\frac{1}{(N)(N)} \sum_{i=1}^{N} \sum_{j=1}^{N}\left[X_{i j}-X_{i j}^{\prime}\right]^{2}
$$

Where, $\mathrm{Nx} \mathrm{N}$ is the total number of pixels $X_{i j}$ and $X_{i j}^{\prime}$ are the pixel values in the original and reconstructed image. The peak to peak signal to noise ratio (PSNR in $\mathrm{dB}$ ) is calculated as:

$$
P S N R=10 \log _{10} \frac{255^{2}}{M S E}
$$

The compression ratio (CR) [7] is another frequently used measure of how effectively an image has been compressed. It is simply the ratio of the size of the image file before and after compression. It is equal to the ratio of bit-rates, in bits/pixel, before and after compression. Since the initial bit-rate is usually 8 bits/pixel, and the entropy $h$ is our estimate of the compressed bit-rate, the compression ratio is estimated by $8 / h$ , where

$h=-\sum_{i} P_{i} \log _{2} P_{i}$ and $C R=8 / h . \quad$ PSNR and CR are presented in table 1 .

From above table we notice that improvement in quality of Lena image compression increased from 17.223 to 19.002, in both of Mohammed and Peppers images the quality and compression ratio increased as well. 
Table IQuality and Compression Raito for standard and Proposed Algorithm

\begin{tabular}{l|l|l|l|l}
\hline \hline \multirow{2}{*}{ Image } & \multicolumn{2}{|c|}{ Standard Quantization } & \multicolumn{2}{l}{ Proposed Algorithm } \\
\cline { 2 - 5 } & PSNR & CR & PSNR & CR \\
\hline Lena & 29.274 & 17.223 & 29.767 & 19.002 \\
Mohamed & 29.695 & 17.221 & 30.136 & 18.716 \\
Penners & 29.223 & 18.064 & 30.022 & 19.526
\end{tabular}

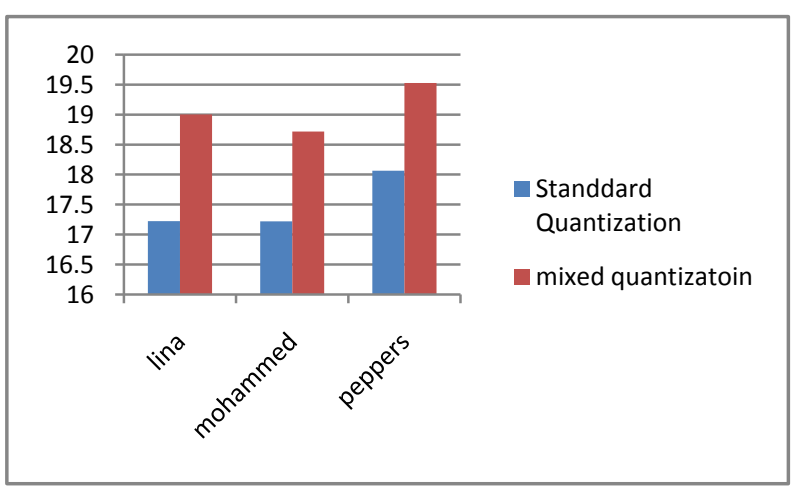

Figure6: Impact of Quantization on Compression Ratio

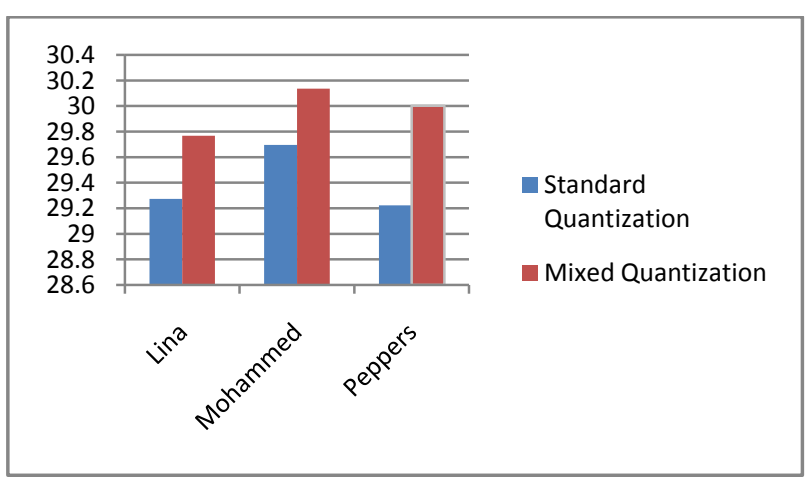

Figure.7 : Impact of Quantization on PSNR

\section{CONCLUSION AND FUTURE WORK}

In this paper, we have proposed a new efficient algorithm for gray images compression using different quantization and compared it with using one quantization. And we found it's give us high compression ratio and high quality. We are working now to perform this algorithm on color image.

\section{REFERENCES}

[1] N. Ahmed, T. Natarajan, and K. R. Rao, "Discrete cosine transform," IEEE Transactions on Computers, vol. C-32, pp. 90-93, Jan. 1974.

[2] W. B. Pennebaker and J. L. Mitchell, "JPEG - Still Image Data Compression Standard,'Newyork: International Thomsan Publishing, 1993.

[3] G. Strang, "The Discrete Cosine Transform," SIAM Review, Volume 41, Number 1, pp.135-147, 1999.

[4] A. K. Jain, "Fundamentals of Digital Image Processing," New Jersey: Prentice Hall Inc.,1989.

[5] A. C. Hung and TH-Y Meng, "A Comparison of fast DCTalgorithms," Multimedia Systems, No. 5 Vol. 2, Dec 1994.

[6] R. C. Gonzalez and P. Wintz, "Digital Image Processing,” Reading. MA: Addison-Wesley,1977.

[7] Khalid S. Introduction to data compression. San Francisco: Elsevier; 2006.

[8] Bonnie L. Stephens, Student Thesis on "Image Compression Algorithms", California State University, Sacramento, August 1996

[9] Gregory K. Wallace, "The JPEG Still Picture Compression Standard", Submitted for publication in IEEE Transactions on Consumer Electronics, December 1991

[10] S. V. Viraktamath, Girish V. Attimarad, “ Impact of Quantization Matrix on the Performance of JPEG", International Journal of Future Generation Communication and Networking Vol. 4, No. 3, September, 2011

[11] ISO/IEC 10918-1, "Digital compression and coding of continuous-tone still images : Requirements and guidelines," Feb., 1994.

[12] Mamta Sharma,\| Compression Using Huffman Coding\|, IJCSNS International Journal of Computer Science and Network Security, VOL.10 No.5, May 2010,pp 133-141

[13] Lakhani, G, Modified JPEG Huffman coding, IEEE Transactions Image Processing, 12(2),2003 pp. 159 169. 